\title{
Kangaroo Mother Care in the Community: Practices in Southern Rwanda
}

\author{
Leonard Ntwari Nyagasare ${ }^{1 *}$, Louise Cauwelier ${ }^{2}$, Assumpta Kayinamura Mwali³, Luk Cannoodt ${ }^{1}$ \\ ${ }^{1}$ NGO Umubano-Impore, Huye, Rwanda \\ ${ }^{2}$ Universiteit Gent Faculteit Geneeskunde en Gezondheidswetenschappen, Management and Policy of Health Care, Ghent, Belgium \\ ${ }^{3}$ Intrabealth, Department of Pediatrics, Kigali, Rwanda
}

*Corresponding author: Leonard Ntwari Nyagasare, Umubano-Impore, Taba, Huye, Rwanda. Email:ntwarileonard@gmail.com

\begin{abstract}
Background

The World Health Organization (WHO) guidelines recommend practicing KMC (Kangaroo Mother Care) continuously for weeks after discharge of preterm newborns. However, little is known about KMC practices in the community in Rwanda and other African countries. Therefore, this study sought to assess KMC practices in Southern Rwanda, primarily after hospitalization and identify barriers to KMC in the community.

Methods

A cross-sectional study was performed with data collected through a survey among 124 caregivers of preterm infants and Community Health Workers. The Statistical Package for the Social Sciences SPSS version 22 was used to analyze the data.

Results

Among all caregivers interviewed, 86.7\% confirmed that they practiced KMC, both in the hospital (KABUTARE District Hospital) as well as after discharge, but there is a large variation in practice time and place. KMC is practiced more during daytime. Working in the fields and lack of support to the caregivers are reported most frequently as barriers to practice KMC in the community.

\section{Conclusion}

KMC-practice is still sub-optimal in Rwanda. Special attention should be directed towards KMCpractice at night and towards the reported obstacles of practicing KMC in the community, e.g. working in the field, lack of support and equipment, difficulties to sleep and health problems. Rwanda J Med Health Sci 2019;2(3):245-251
\end{abstract}

\section{Keywords: Kangaroo Mother Care - Follow-up - Preterm infants - Rwanda.}

\section{Introduction}

WHO defines preterm birth as "babies born alive before 37 weeks of pregnancy or 259 days of gestation”.[1] According to Gates B et al., 15 million babies are born prematurely every year worldwide. Over $60 \%$ occurs in Africa and South Asia.[2]Blencowe $\mathrm{H}$, et al. reported that prematurity is the leading cause of newborn deaths and the second-leading cause of death in children under the age of five, after pneumonia.

In low-income countries, gestational age is often not registered. In that case, LBW tends to be used as a proxy for preterm birth, because preterm births often result in low birth weight (LBW). WHO defines LBW as "weight at birth of less than 2,500 grams".[2]

Beck et al. state that the morbidity associated with preterm births often extends to later life, resulting in enormous physical, psychological and economic costs. [3] Thus, interventions that reduce infant mortality and morbidity and the resulting economic costs related to it would be an important advance in health care.

According to Charpak et al. a feasible, cost-effective solution can be found in Kangaroo Mother Care (KMC). The method has been reviewed and adjusted, resulting in a new model consisting of three main components: position, feeding and follow-up policies. The position demands a continuous skin-to-skin contact between adult and infant, the latter only wearing a diaper, socks and a cap. Furthermore, the newborn must be held in a vertical position until he can regulate his own temperature. When possible, the child must exclusively be breastfed. Fortification (supplements) is allowed if there is a poor weight gain $(<15 \mathrm{~g} / \mathrm{kg}$ per day).[4]

Akhtar et al. state that KMC provides an alternative to incubator care, without separation from the mother.[5] As stated by Conde-Agudelo and Bergh et al., it is proven to reduce mortality, severe infections, hypothermia and length of hospital stay and to increase weight gain and breastfeeding. However, the implementation 
of KMC is often varied.[6,7] According to the World Bank this kind of care is especially recommended in low-income and lower middle-income countries, respectively characterized by an economy with a Gross National Income (GNI) per capita of $\$ 1,045$ or less, and economies with a GNI per capita of $\$ 1,046$ to $\$ 4,125$. [8] Once the baby is medically stable and the caregiver is familiar with the KMC-method and in control of the breathing-sucking-swallowing coordination, the infant can be discharged. However, this assumes that an appropriate follow-up system is in place and that the caregiver of the preterm infant is willing to collaborate with this program. If this is not sufficiently practiced, the infant runs elevated risks of morbidity, malnutrition, development delays and even death. WHO recommends that $\mathrm{KMC}$ is practiced continuously until the baby weights 2,500 g. or until 40 weeks after gestation. Most preterm babies gain between 15 and 30 grams a day.[9] It means that KMC should be practiced at least three to four weeks (day and night) after hospital discharge when the baby is discharged at $2.000 \mathrm{~g}$. or less. In some countries, such as Rwanda, LBW babies are often discharged at, or even before reaching, 2,000 g.

\section{Methods}

\section{Study design}

We utilized a cross-sectional study design to assess KMC, among LBW infants who received neonatal care at KABUTARE District Hospital (DH) in Southern Rwanda. The study also included the infants' caregivers.

\section{Study site}

The study was conducted in KABUTARE DH, where a neonatal medium-care unit was introduced in August 2010. Since January 2011, mothers of preterm newborns are offered the opportunity to get used to the KMC-method by staying for one or more days in a well-equipped KMC-ward. Once the infant weighs 2,000 grams, mother and child can be discharged. However, if the mother has a lot of work or has other children at home to take care of, discharge can be arranged if the infant weighs at least 1,800 grams.

KMC was first introduced in Rwanda in 2008 by the WHO. This article reports on KMC-practices in KABUTARE DH and after discharge from that hospital, with emphasis on KMC after discharge.

\section{Follow-up}

Once the mother (or caregiver) returns home, the Community Health Workers (CHWs) make a follow-up. For the preterm/LBW infants, CHWs are supposed to visit these families at home three times during the first four weeks, of which two times during the first week. These CHWs are best placed to observe KMC-practices after hospital discharge and it was for this reason that they were involved in the study.

\section{Study population}

The inclusion criteria for the study were: (A) low birth-weight infant $(<2,500$ gram), (B) born between 01/01/2011 and 31/12/2012, (C) received neonatal care in KABUTARE DH, (D) discharged from that hospital alive, (E) discharged directly to the home of the caregiver (mostly the mother).

For each child included in the study that could be reached, both the caregiver and the $\mathrm{CHW}$, who is responsible for maternal and child health in the village of the child's family, were interviewed.

Infants who were transferred to the University Teaching Hospital of BUTARE (CHUB) to receive neonatal intensive care were excluded.

\section{Sample size}

The original database consisted of $185 \mathrm{LBW}$ children. Of these, 30 children died after they were discharged to home from KABUTARE DH. Thirty-one families could not be reached, either because they moved outside the area or because the address (village) in the hospital files was incomplete or incorrect. The remaining 124 children were visited at their homes.

The 124 mothers or caregivers of these children as well as their 124 respective CHWs were interviewed. Participation was voluntary and all participating mothers and CHWs signed the informed consent form.

\section{Data collection procedure}

This study was conducted between 2014 and 2015.The data were collected through a quantitative survey with some open-ended questions. A survey questionnaire was developed in English by the principal investigator and approved by the Research Committee within the Faculty of Public Health and Human Nutrition, Catholic University of Rwanda. The questionnaire was divided into three sections. Section 1: Background information on child and household. Section 2: Background information about the interview, such as address and date of the interview. Section 3: Information from the caregiver and the CHW who knows the family best.

The questionnaire was then translated in the local language, Kinyarwanda. The interviews were conducted with the mother of each selected child. The interview was done in two parts. The first part was addressed towards the caregiver and the second part towards the Community Health Worker.

\section{Statistical analysis}

The analysis of the data was performed through SPSS Version 22. Each variable was thoroughly checked by running the frequencies. All the answers of the open ended questions were given a code. We conducted a descriptive analysis of all the answers (frequencies of each code). 


\section{Ethical considerations}

This study was approved by the Rwanda National Ethics Committee. All the interviews were conducted after signing an informed consent form. In order to treat the gathered data anonymously, no name or address of the respondents, nor of any institution, was shared with any other person than the researcher for data compilation. Authorization was also requested and granted from the local authorities, Kabutare DH, the Health Centers (HCs) and the head of the Community Health Workers of each sector.

\section{Results}

\section{KMC practice in the hospital}

The findings show that $82.3 \%$ respondents received information about the KMC method in the hospital. The majority received the information between 12 hours and 1 day after delivery (21\%) or between 3 and 7 days (21\%). Others received it between 1 and 12 hours after delivery (18\%), between 1 and 3 days (14\%), less than 1 hour $(10 \%)$. There was a large variation in the timing of receiving this information, going from less than one hour after delivery $(10 \%)$ to more than two weeks after delivery (9\%)(figure 1).

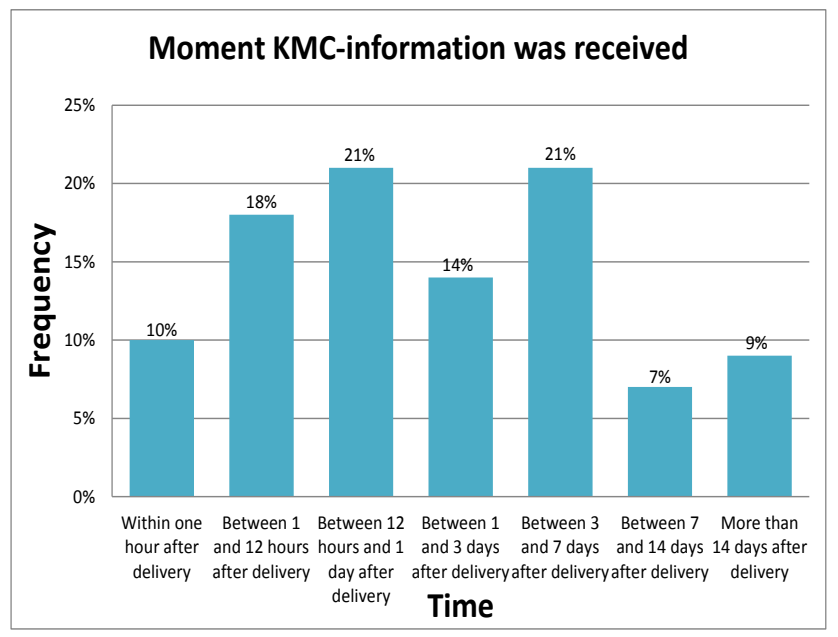

Figure 1. Moment KMC information was received $(\mathrm{N}=100)$

The large majority of the interviewe (91.1\%) confirmed that the KMC-practice started in the hospital. In six cases, it was not the mother who practiced KMC in the hospital.

There was also a large variation in the time that the persons concerned started using KMC in the hospital. Some already started within one hour after delivery $(18.6 \%)$, while a small number $(7.1 \%)$ started only one month after delivery (see Table 1).
Table 1. Moment that KMC started in the hospital (N=113)

\begin{tabular}{lll}
\hline Variable & n (\%) & $\begin{array}{l}\text { Cumu- } \\
\text { lative \% }\end{array}$ \\
\hline
\end{tabular}

Within one hour after delivery

21 (18.6)

18.6

Between 1 and 12 hours after delivery

$19(16.8)$

35.4

$>12$ hours and $<$ one day after delivery

$15(13.3)$

48.7

Between 1 and 3 days after delivery

$17(15.0)$

63.7

Between 4 and 7 days after delivery

$20(17.7)$

81.4

Between 8 and 31 days after delivery

$>$ One month after delivery

$8 \quad(7.1)$

100.0

Not all women who delivered, started KMC in the hospital. In fact, $8.9 \%$ of them did not start KMC in the hospital. Of those who did start KMC in the hospital, $85 \%$ stayed at least one day in the KMC-room. Of those who stayed in the KMC-room, $32.3 \%$ left the room within one week, while $27.1 \%$ stayed there one month or more.

\section{KMC practice in the community}

Starting KMC in the hospital is the best predictor for practicing $\mathrm{KMC}$ in the community. In fact, no one started KMC after hospital discharge, while only 5.3\% of the main respondents, who reported that they started KMC in the hospital, did not continue KMC after hospital discharge. In total, $86.7 \%$ of interviewed persons who practiced $\mathrm{KMC}$ in the hospital reported to have continued $\mathrm{KMC}$ at least one day in the community (= after discharge). Of them, $83.7 \%$ did so during at least one night. So, there is some difference between the KMC-practice during daytime and at night.

Of those who practiced KMC in the community during daytime, $8.2 \%$ practiced it up to one week, 49 $\%$ practiced it between one week and four weeks, while $42.8 \%$ practiced KMC more than 4 weeks.

Of those who practiced $\mathrm{KMC}$ in the community during daytime, $42.7 \%$ did so only 1 to 3 hours per day, while $27 \%$ did so for more than 6 hours per day.

Of those who practiced KMC at home during the night, all but two gave the duration that they continued doing so. Among those, $23.8 \%$ stopped before the end of the first week, while $28.7 \%$ continued during one month or more.

Of those who continued KMC at night, almost half did so only one to three hours per night, while about $1 / 4 \mathrm{did}$ so the whole night (more than nine hours a night).

Figure 2 indicates who faced some obstacles during KMC practice. In fact, among 100 caregivers practicing $\mathrm{KMC}, 72 \%$ of them indicated that they did experience obstacles. 


\section{Difficult to continue KMC at home}

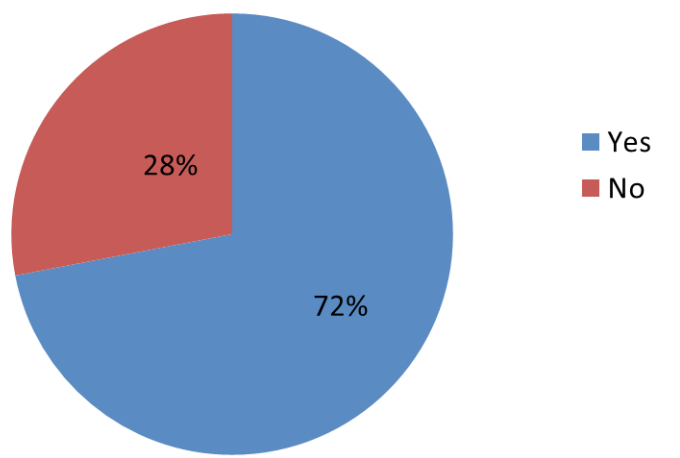

Figure 2. Difficult to use KMC at home $(\mathrm{N}=100)$

Table 2 gives more clarification of why KMC is not practiced more frequently in the community $(80.6 \%$ of main respondents answered this question).

Each respondent could give one or more obstacles/ difficulties. Of those who did mention at least one barrier/obstacle, about $71 \%$, mentioned that it is difficult to work when practicing KMC (most of them specified 'working in the fields'), almost $53 \%$ mentioned that it is difficult to get food, $43 \%$ mentioned lack of equipment, and one fourth mentioned health problems (see table 2 for a complete overview of barriers). The frequency can also be expressed in percentage of all caregivers interviewed. 'Difficult to work' was mentioned by $41 \%$ of all caregivers, 'difficult getting food' was mentioned by $30.6 \%$ and 'lack of equipment' by $25 \%$ of all main respondents. Among main respondents, 11.3 $\%$ reported 'having twins' as a barrier. Of those, 85.7 $\%$ had both twins alive at the moment of the interview. Two other mothers with both twins alive did not report this as a barrier. Fourteen mothers have lost one of both twins before the interview took place. Only one of them reported that having twins was a barrier for practicing KMC. In one case the barrier 'twins' was mentioned by a mother with a singleton child in the sample.

Table 2. Obstacles in practicing KMC reported by the caregivers $(\mathrm{N}=72)$

\begin{tabular}{lr}
\hline Variable & $\mathbf{n} \mathbf{( \% )}$ \\
\hline Difficult to work (in the fields) with KMC & $51(70.8)$ \\
Difficult getting food & $38(52.8)$ \\
Lack of equipment such as warm clothes, a mattress, and sheets. & $31(43.1)$ \\
Health problems such as HIV, back pain and other sicknesses. & $18(25.0)$ \\
Having twins & $14(19.4)$ \\
Difficulties to carry the baby on the chest & $10(13.9)$ \\
Difficulty to sleep & $9(12.5)$ \\
Disagreements with the partner & $8(11.1)$ \\
Having a disability & 2 \\
\hline
\end{tabular}

Note: Several caregivers mentioned more than one obstacle. N gives the number of caregivers who mentioned the specified types of obstacles.

\section{Improving KMC-practice in the community}

Both the main respondents and the CHWs were asked to give some suggestions about how the practice of KMC in the community can be improved. In both cases, each respondent could give more than one suggestion. The answers are quite different, so they are reported in two tables. Table 3 presents the different suggestions made by the caregivers.

Table 3 indicates that most of the caregivers suggest that they should get more support. This includes more support in the provision of food, health insurance, equipment to carry the baby and to keep the baby warm, as well as to give birth in health facilities rather than at home. A second set of suggestions refers to the responsibilities of the mothers/caregivers. According to some caregivers, they should follow the instructions of the CHWs better, have more patience, make more time for feeding and caring for the baby and avoid activities that make it difficult to practice KMC. A third set of suggestions refers to responsibilities of healthcare personnel (visit the mothers at home, inform the families more). 
Table 3. Suggestions that can improve future KMC practice in the community, as reported by the caregivers $(\mathrm{N}=124)$

\begin{tabular}{lc}
\hline Variable & n (\%) \\
\hline Caregivers need more support in the provision of food & $30(24.2)$ \\
Caregivers and family members need to be better informed & $26(21.0)$ \\
Caregivers need more support in the provision of insurance & $24(19.4)$ \\
Caregivers need more support in the provision of equipment to keep warm. & $21(16.9)$ \\
Caregivers should spend more time taking care of the baby & $18(14.5)$ \\
Caregivers need to better follow the instructions given by the CHW & $14(11.3)$ \\
Caregivers need more support in the provision of modern carrying pouches. & $9(7.3)$ \\
Caregivers need to better feed the baby & $7(5.6)$ \\
Healthcare staff should visit the mothers at home & $6(4.8)$ \\
Mothers need to be encouraged to give birth at a health facility & $4(3.2)$ \\
The caregivers need to have more patience & $2(1.6)$ \\
Caregivers should not do difficult activities & $2(1.6)$ \\
\hline
\end{tabular}

The suggestions made by the interviewed CHWs to improve future $\mathrm{KMC}$ practice in the community are shown in table 4. The CHWs suggest several items that were not mentioned by the caregivers, while they did not suggest items mentioned by the caregivers. Only support in accessing food and need to explain the benefits of $\mathrm{KMC}$ was suggested frequently by both types of respondents. Several CHWs also referred to the importance of involving others, including health centers, to support KMC and to care for LBW infants after discharge from the hospital. Some CHWs stressed the importance of hygiene for the mother and of practicing KMC during the night (see table 4).

Table 4. Suggestions that can improve future KMC practice in the community, as reported by the CHWs $(\mathrm{N}=124)$

\begin{tabular}{|c|c|}
\hline Variable & n $\quad(\%)$ \\
\hline Support vulnerable caregivers in the access to food during KMC & $42(33.9)$ \\
\hline Keep explaining the benefits of KMC to caregivers and family & $30(24.2)$ \\
\hline Provide equipment for the caregivers such as sheets, a mattress, and warm clothes. & $27(21.8)$ \\
\hline Keep the baby in warm conditions using a hat and socks & $18(14.5)$ \\
\hline Ask and advice others to support the caregivers during KMC & $15(12.1)$ \\
\hline Provide health insurance for mothers using KMC & $14(11.3)$ \\
\hline Provide modern carrying pouches & $13(10.5)$ \\
\hline Provide more training for the CHWs & $12(9.7)$ \\
\hline Empower HCs to care for LBW infants at their level & $10(8.1)$ \\
\hline Caregivers need to take care of their hygiene & $(7.3)$ \\
\hline Bring the child to check-up & $(6.4)$ \\
\hline Get help when the child has some problems & $4(3.2)$ \\
\hline Practice it more at night & $3(2.4)$ \\
\hline
\end{tabular}

\section{Discussion}

There is hardly any information published about the practice of KMC after hospital discharge of LBW babies in sub Saharan countries. This study revealed that some caregivers did not continue KMC after discharge. Only $86.7 \%$ did so for at least one day, only $73 \%$ did so at least one night. Also, the average number of days and nights that they practiced KMC were likely to be suboptimal in most cases. One study from Nguah et al. reported that in Ghana $95.5 \%$ of the mothers planned to continue KMC after hospital discharge during the day and 93.1\% were willing to practice it during the night.[10] The same study indicated that $61.9 \%$ of 202 mothers in Ghana thought $\mathrm{KMC}$ was easy to practice in the community. [10] The results of this study show that only $22.6 \%$ of all caregivers experienced no difficulties in the practice 
of KMC after hospital discharge. It would be interesting to analyze why the results differ in both countries and to conduct similar studies in other sub Saharan countries.

This study also focused on the barriers for practicing KMC in the community. The most frequently reported barrier is the difficulty to work (in the fields) while simultaneously practicing KMC (70.8\% of those caregivers that reported KMC barriers). According to several published studies, the interference of KMC in the mother's daily work schedule is a frequently reported barrier.[4,11-13] It is noteceable that this study was conducted in a rural area of Rwanda. This is also visible through the reported profession, $89.5 \%$ said to be farmer, cultivator or planter. Rural women may choose to stop or interrupt the KMC practice as it is common practice in the sub-Saharan region to work in the fields with the baby on the back. Bergh and Bazzano et al. also reported this tradition in studies conducted in Ghana, Malawi, Mali and Uganda.[7,11]

This study also focused on suggestions that may improve the use of KMC in the community. There are clear differences between the caregivers and the CHWs concerning the different types of suggestions and their frequencies. Both groups often mentioned supporting caregivers in provision of food, explaining to the family the benefits of $\mathrm{KMC}$, and providing equipment to keep the baby warm. Caregivers often mentioned that they can do better in caring for the baby, while CHWs also suggested that the CHWs and the HCs need more training to care for LBW infants at their level. Several CHWs also reminded about the importance of using hat and socks by the babies and taking care of the hygiene of the caregivers.

Some barriers and suggestions are comparable to what is reported in other published studies. According to Bergh et al. it is beneficial to have support from the partner during KMC.[14,15] This study reports that $11 \%$ of the 72 caregivers see 'disagreement with their partner concerning KMC' as one of the barriers.

The findings in this study show that difficulties to sleep were reported by $12.5 \%$ of the caregivers as a challenge for practicing KMC at night. It has also been shown by Charpak \& Ruiz-Pelaez that this should be one of the main concerns before starting KMC. [4] The same authors also mentioned that mothers experience difficulty to take care of their own hygiene. [4] The same issue was reported by $7.3 \%$ of the CHWs in this study as an issue to be dealt with in order to improve the practice of KMC. However, the caregivers in this study did not mention it as a barrier in relation to KMC practice. Education about basic hygiene could help dealing with this issue.

There were a few limitations to this study. A first limitation is memory bias due to the time between the date of birth of the child and the time when the interviews were conducted. All children were born between January $1^{\text {st }} 2011$ and December 31 ${ }^{\text {st }} 2012$, however the interviews only started in June 2014. As a result, the received information could be less accurate than when the interviews would have been conducted in an earlier period of time.

Not all caregivers were present in the hospital at the time the child received neonatal care. This may explain 'missing values' for some answers of the questionnaire. Lastly, a large majority of caregivers were living in the district of Huye. The other caregivers interviewed were living in two neighboring districts. The results are, therefore, not necessarily representative for Rwanda as a whole.

\section{Conclusions and recommendations}

This study sought some answers to three research questions: "To what extent and how long has the KMC method been applied in the hospital and after discharge?", "What barriers have been identified concerning the practice of KMC in the community?" and "What suggestions can be proposed to improve future KMC practice in the community?"

KMC started in January 2011 in Kabutare distric hospital. Since then, a large majority of LBW newborns who receive neonatal care in that hospital also receive KMC during their hospital stay and after they are discharged to go home. However, the responses of 124 caregivers showed that there is a large variation in the timing at which they received information about KMC, the number of days that the caregivers stayed in the $\mathrm{KMC}$ room, the number of days and nights that KMC was practiced in the community, as well as the number of hours per day and per night.

The results of this study indicate clearly that KMC practice is not yet optimal, especially after hospital discharge. Some caregivers stopped KMC too early to be sufficiently beneficial or did not even start KMC during the night. Only a minority practiced KMC continuously, day and night. Therefore, it can be recommended that all health care providers would scale up their efforts to provide information (concerning the benefits of KMC and how to practice it) continuously to all mothers giving birth to LBW children, as well as their partners. Special attention should be directed towards KMC practice at night as $\mathrm{KMC}$ appears to be less commonly practiced then.

Many respondents reported difficulties to practice KMC in the community. The answers suggest that several difficulties can be overcome by providing more support to the caregivers in getting food, health insurance, and material that help them to practice KMC. As "working 
in the fields" is the most frequently mentioned obstacle to practice KMC in rural areas, specific strategies have to be worked out to deal with this obstacle.

It is recommended that the CHW-coordinators of the health centers would supervise the CHWs more closely and give the necessary training to be sure that they are all aware of the importance of more frequent home visits for preterm infants, and of continuing these visits for a longer period after birth. This also requires that the health centers are well informed by the hospital immediately after each discharge of a preterm neonate. This study is limited to the experience in a few districts in the South of Rwanda, the large majority living in one district (Huye). As it is the first in Rwanda and surrounding countries, it brings important new information about the post-neonatal care to LBW-infants. Preferably, this study should be repeated in other districts and other subSaharan countries on a larger scale and at different times after the children are discharged from the hospital (six months, one year, etc...) to collect additional evidence to support policy-makers, health care providers and health service researchers.

\section{Limitation of the study}

It should be pointed out that this research did not attempt to analyze the real causes of suboptimal use of $\mathrm{KMC}$ in the community

\section{Competing interests}

The authors declare that they have no competing interests.

\section{Authors' contributions}

LNN coordinated the field work and data analysis.

AMK provided guidance for the medical and KMCpractice aspects of the project.

LC supervised the research project

\section{Acknowledgements}

We acknowledge the Rwanda National Ethics Committee for approving this study, and the NGO UMUBANOIMPORE (IMPORE means "Improving Maternal and Pediatric Outcome: the Rwandan Experience"). We also acknowledge Dr. Louis Ide (who financed this project under the name August10) and the Catholic University of Rwanda also funded part of this project (under the name FUAPI).

This article is published open access under the Creative Commons Attribution-NonCommercialNoDerivatives (CC BY-NC-ND4.0). People can copy and redistribute the article only for noncommercial purposes and as long as they give appropriate credit to the authors. They cannot distribute any modified material obtained by remixing, transforming or building upon this article. See https://creativecommons.org/ licenses/by-nc-nd/4.0/

\section{References}

1. Gates B, John G, Blencowe H, Cousens S, Chou D, Oestergaard MZ, et al. 15 million preterm births : priorities for action based on national, regional and global estimates.who.int/pmnch/media/ news/2012/borntoosoon_chapter2.pdf

2. Leung C. Born too soon. Neuro Endocrinol Lett [Internet]. 2004;25:133-136. Available from: http:// www.ncbi.nlm.nih.gov/pubmed/15735596.

3. Beck S, Wojdyla D, Say L et al. The worldwide incidence of preterm birth: a systematic review of maternal mortality and morbidity. Bull World Heal Organ. 2010;88:31-38.

4. Charpak N, Ruiz-Pelaez JG. Resistance to implementing Kangaroo Mother Care in developing countries, and proposed solutions. Acta Paediatr. 2006;95:529-34.

5. Akhtar K, Haque M KS. Kangaroo Mother Care : A Simple Method to Care for Low- Birth-Weight Infants in Developing Countries. 2013;5:49-54.

6. Conde-Agudelo AB-R. Kangaroo Mother Care to reduce morbidity and mortality in low birth weight infants (Review). Cochrane Database Syst Rev. 2011;3.

7. Bergh, A.M., Kerber, K., Abwao, S., de-Graft Johnson, J., Aliganyira PD et al. Implementing facility-based Kangaroo Mother Care services: lessons from a multi-country study in Africa. BMC Heal Serv Res. 2014;14:293.

8. Agénor PR. Caught in the Middle? The Economics of Middle-Income Traps. Journal of Ecomic Surveys 2017; 31(3):771-791.

9. WH. O. Kangaroo mother care: a practical guide. WHO Geneva. 2003;25-30.

10. Nguah, S.B., Wobil, P.N.L., Obeng, R., Yakubu, A., Kerber KJL et al. Perception and practice of Kangaroo Mother Care after discharge from $h$. BMC Pregnancy Childbirth. 2011;11:1-8.

11. Bazzano, A., Hill, Z., Tawiah-Agyemang, C., Manu A ten A. Introducing home based skin-to-skin care for low birth weight newborns: a pilot approach to education and counseling in Ghana. Glob Heal Promot. 2012;19:42-9.

12. Hunter, E.C., Callaghan-Koru, J.A., Mahmud, A.A., Shah, R. FA et al. Newborn care practices in rural Bangladesh: Implications for the adaptation of kangaroo mother care for community- based interventions. Soc Sci Med. 2014;122:21-30.

13. Parikh, S., Banker DS \&Bala D. Barriers in implementing community based Kangaroo Mother Care in lowincome community. J Med Sci. 2013;2:36-8.

14. Bergh, A.M., Charpak, N., Ezeonodo AU van R. Education and training in the implementation of kangaroo mother care. South African J Child Heal. 2012;6:38-45. 